\title{
Impact Analysis on Go-Kart Chassis with Variable Speeds using Ansys 19.0
}

\author{
Pnv Balasubramanyam, B. Nageswara Rao, Moon Banerjee, B. Lakshmana Swamy
}

\begin{abstract}
The main aim of this paper is to model the go kart chassis in solid works and perform the impact analysis of the motor vehicle chassis in Ansys. The purpose of impact analysis is to study the behaviour of the chassis during impact on the go-kart chassis. The National Go Kart Racing is the one of the ways which provide the platform for doing innovations and showing creativity of students. The motor vehicle chassis is completely different from standard automobile chassis. The basic requirements of the chassis will be less weight and more strength. The material that we opted for this chassis is AISI 4130 which is a medium carbon steel having good tensile strength and better machinability and offers good balance to toughness and ductility. Such that by performing impact analysis at different impact speeds we are going to analyses the chassis behaviour for the given conditions. The chassis frame is backbone of vehicle it should be able to with stand different types of loads that are developed during vehicle at rest and in motion. The given chassis is tested under different impact conditions and the results such as deformations, stresses are determined using ANSYS 19.0 software.
\end{abstract}

\section{INTRODUCTION:}

American Art Ingles is generally accepted to be the father of karting. The primary kart was done in Southern California in 1956. Now Karting rapidly spread to other countries and currently has a large following in Europe. The first kart manufacturer was AN yank company, Go Kart Manufacturing Co. (1958). In 1959, McCulloch was the primary company to provide engines for karts. Its initial engine, the McCulloch MC-10 was an adapted chainsaw two-stroke engine. Go- kart was invented in California by art angles and Lou Borelli using 100 cc motor engines and strong steel frames. Then newly designed karts were popular in the year 1959-1960. Its main parts are the chassis made up of steel pipes that are welded to gather. Go-kart Racings is a cheaper and smaller way of automobile racing and lot of safety compared to other motor racing sports such as formula one. Today kart racing is not only anticipated by adults but the younger generations.

Revised Manuscript Received on October 30, 2019

* Correspondence Author

P. N. V. BALA SUBRAMANYAM, Research Scholar, Department of Mechanical Engineering, Koneru Lakshmaiah Educational Foundation, Vaddeswaram, Guntur, Andhra Pradesh, India

B. NAGESWARA RAO, Professor, Department of Mechanical Engineering, Koneru Lakshmaiah Educational Foundation, Vaddeswaram, Guntur, Andhra Pradesh, India

MOON BANERJEE, Associate Professor, Department of Mechanical Engineering, Koneru Lakshmaiah Educational Foundation, Hyderabad, Telengana,India

B. LAKSHMANA SWAMY, Professor, Department of Mechanical Engineering, Koneru Lakshmaiah Educational Foundation, Hyderabad, Telengana,India

(C) The Authors. Published by Blue Eyes Intelligence Engineering and Sciences Publication (BEIESP). This is an open access article under the CC BY-NC-ND license (http://creativecommons.org/licenses/by-nc-nd/4.0/)
The chassis of automotive vehicle should be stable with high torsional rigidity and have comparatively high degree of flexibility as no suspension. And the structure should have enough strength to withstand the Impact load on chassis at different speeds.

The chassis is designed in such a manner, so it can ride safe and smooth the loads that are developed does not affect the structural strength of the chassis. The chassis is the backbone of an automobile and it must as flexible as enough. In automotive structural analysis is the most important processes in developing an extremely good vehicle. FEM analysis software have greatly increased the protection, dependability and luxury environment and manufacturing potency of today's vehicles. The primary concern is driver safety. A vehicle is expected to provide utmost protection to driver in order to prevent accidents. In a well-designed automobile, the automotive body and numerous parts add measure as a protecting layer for the occupants of the vehicle. They serve better protection in absorbing the energy of impact. The common approach involves for designing the prototype and performing the crash tests. This process is time consuming and expensive. The availability of FEA \& FEM has made the researchers to make their work easy \& it will consume less time. The product cycle of a new vehicle has been decreased and theresultant vehicle is safer and better ride quality as the design process is replaced with computer simulation to evaluate the vehicle design.

\section{AUTOMOBILE STRUCTURE:}

Engineers deigns and manufacture the vehicle body structures to resist static and dynamic loads to restrict throughout the vehicle life cycle. It provides most of the vehicle rigidity once in bending and in torsion. The designed chassis will reduce injury when crashes happens. The vehicle body in conjunction with the suspension is intended to reduce vibrations and mechanical noises transfer to the occupants. The chassis structure is designed to maintain withstand loads and provide good safety with protection during crashes or in accidents. The automotive vehicle structure has evolved since the last ten decades to satisfy consumer needs and demands according to their requirements. Currently car body structures designed in light weight and high strength.

\section{GO-KART CHASSIS:}




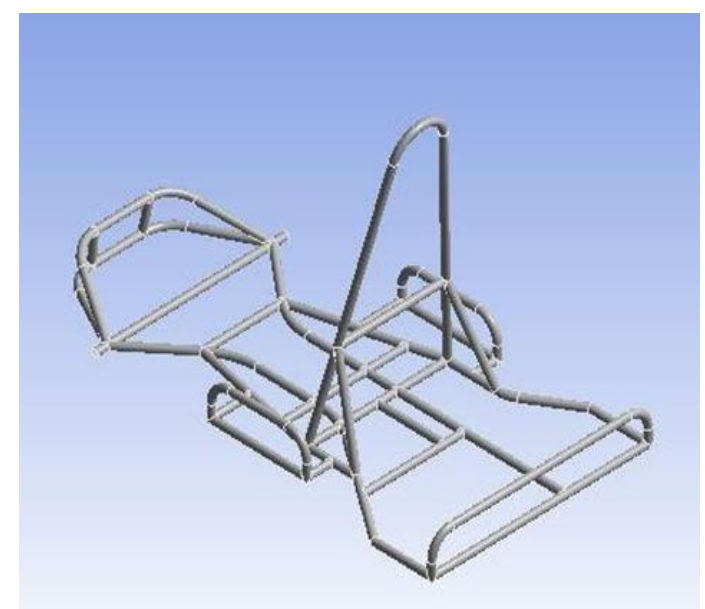

Figure:01

The Go-Kart chassis is a small four wheeled vehicle with no suspension and differential. It is a light powered vehicle which is used for sports racing. The chassis of motor vehicle could be a skeleton frame created and designed of hollow pipes made up of different materials of different cross sections. The chassis of motor vehicle should be stable with high torsional rigidity and have comparatively high degrees of flexibility when there is no suspension. And the structure should have enough strength to withstand the Impact load at differentspeeds. The chassis can carry and support the power train, power unit, running system, etc.

\section{MATERIAL PROPERTIES:}

Mostly used materials are AISI4130(chromo lay steel), AISI1018. Here we have taken material AISI4130 it is best \& lightest one when it comes to making frames, but its cost is expensive than other steels which are available in market.it is a low alloy steel combining of chromium\& molybdenum, and has good strength, toughness \& machinability. It has good fatigue strength and corrosion resistance.

\begin{tabular}{|l|l|}
\hline $\begin{array}{c}\text { MATERIAL } \\
\text { PROPERTY }\end{array}$ & VALUE \\
\hline Ultimate tensile strength & $655 \mathrm{Mpa}$ \\
\hline Poisson's ratio & 0.33 \\
\hline Density & $7.85(\mathrm{~g} / \mathrm{cc})$ \\
\hline Bulk modulus & $140 \mathrm{Gpa}$ \\
\hline Shear modulus & $80 \mathrm{Gpa}$ \\
\hline Elastic modulus & $210 \mathrm{Gpa}$ \\
\hline
\end{tabular}

Crashworthiness tests criteria and model requirements: In the automobile industry Crashworthiness denotes a measure of the vehicle structural ability to withhold deformation and yet provide safety for the occupants in crashes involving sudden impact loading. The vehicle crashworthiness is evaluated in three distinct modes.

- Front Impact crashtest

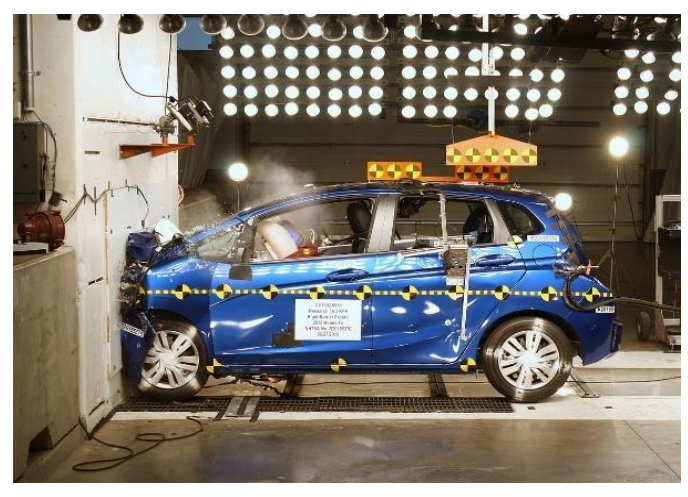

Figure-2

2016 Honda Fit striking a wall head-on at $56 \mathrm{~km} / \mathrm{h}$.

- Driver side (or) Passenger side crash test

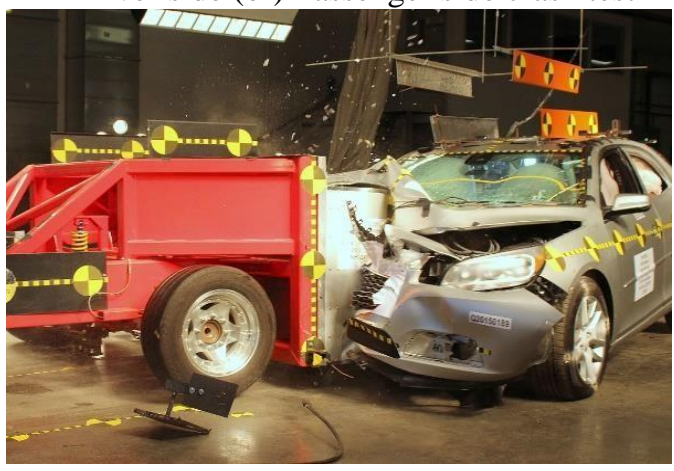

Figure-3

Passenger-side oblique crash test of a $2015 \underline{\text { Chevrolet }}$ Malibu.

- Combined test at front impact and side impacttest

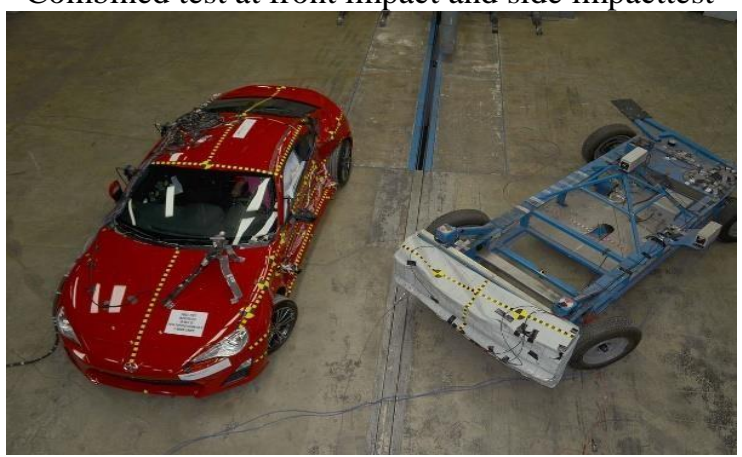

Figure-4

Side impact crash test of a 2014 Scion FR-S.

The above conditions are shown in the Figurestherigidbodyi.e.Stiffness behavioris given to the wall (or) Barrier as weneglect the deformation of the wall (or) Barrier (Figure5.1).thefixedsupportisthenapplied to the wall as it is an immovable object (Figure5.2)andfinallythevelocityisapplied for the chassis and the chassis is constrained in all DOF except $\mathrm{X}$-axis as we had applied the velocity in the X-Direction based on the model (Figure5.3). 


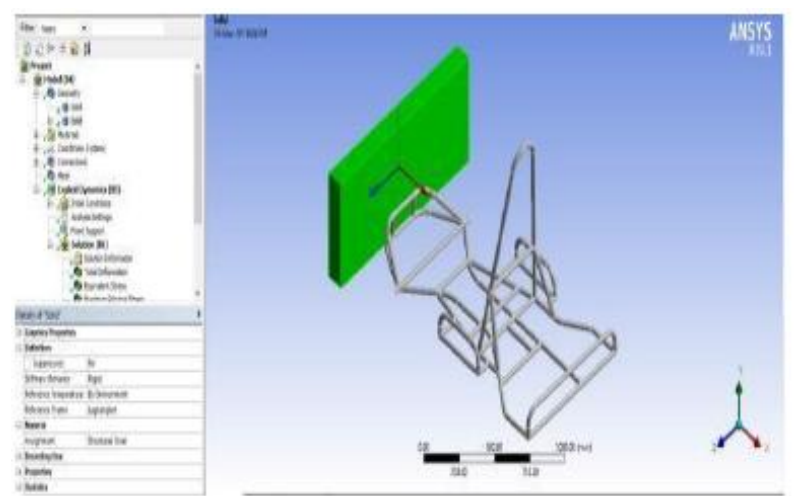

Figure -5.1

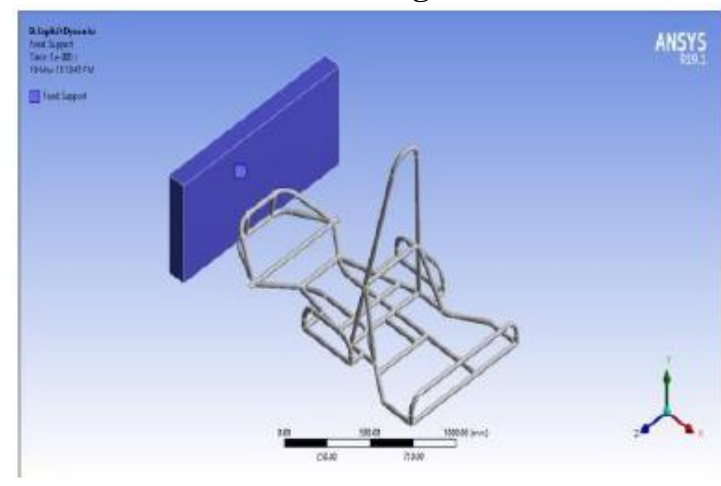

Figure -5.2

The velocity requirements are taken from

-FederalMotorVehicleSafety Standard.

-Insurance Institute for Highway SafetyAnd we have performed impact tests at the following speeds $18 \mathrm{~m} / \mathrm{s}$, $22 \mathrm{~m} / \mathrm{s}, 24 \mathrm{~m} / \mathrm{s}$ and $28 \mathrm{~m} / \mathrm{s}$.

Meshing of Go-kart Chassis:

A fine mesh has been created by using Ansys for better and accurate results. The figures show the mesh of the three different types of crashworthiness tests (or) analysis.
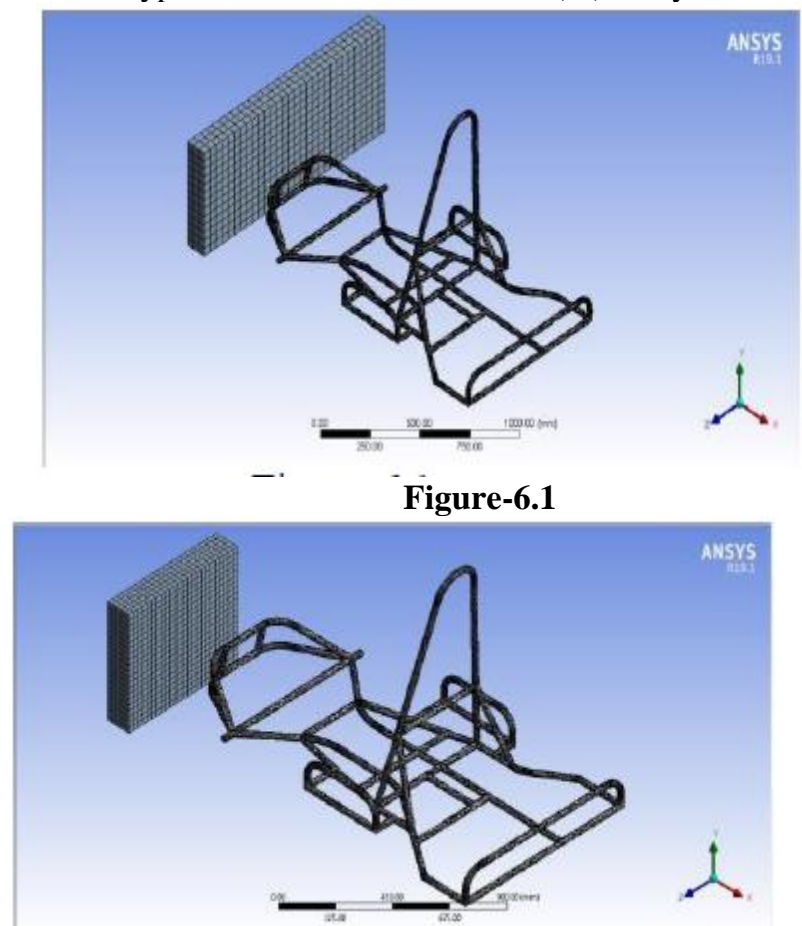

Figure-6.2 \& Figure-6.3

IV. RESULTS AND PLOTS:

Full Frontal Impact Results:

Retrieval Number F8494088619/2019@BEIESP

DOI: 10.35940/ijeat.F8494.088619

Journal Website: www.ijeat.org
Total Frontal impact analysis has been carried out with the help of the Ansys software and after solving the model withthe boundary conditions the results are as follows.

Total Deformed generated in the chassis at different speeds:
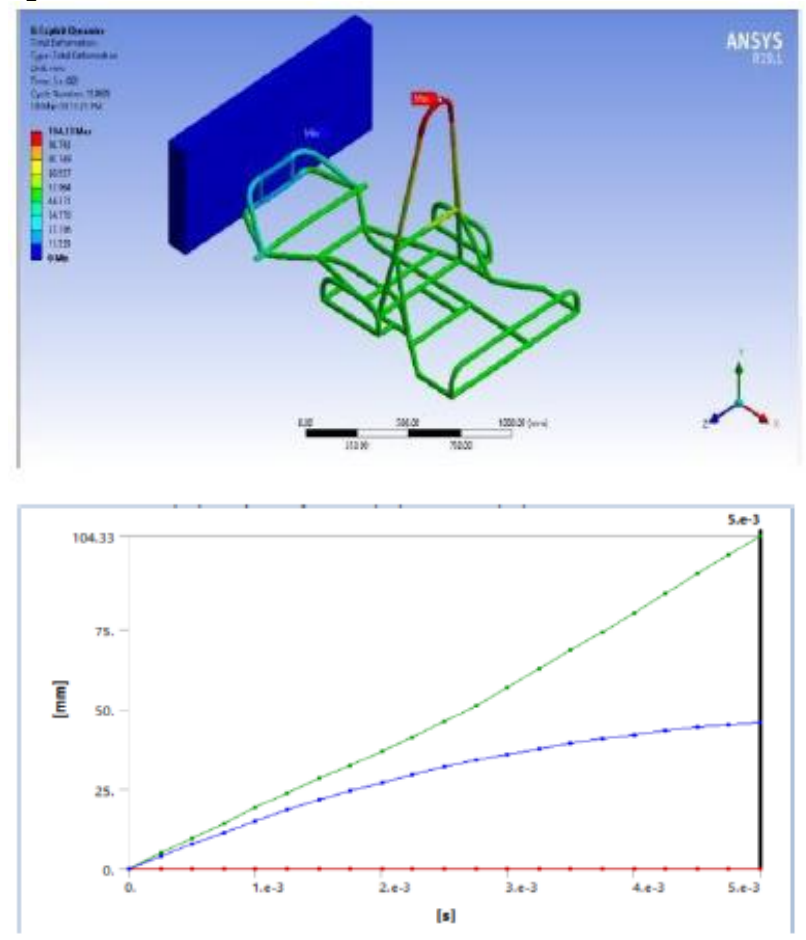

Figure-7 Total deformed chassis at $18 \mathrm{~m} / \mathrm{s}$.
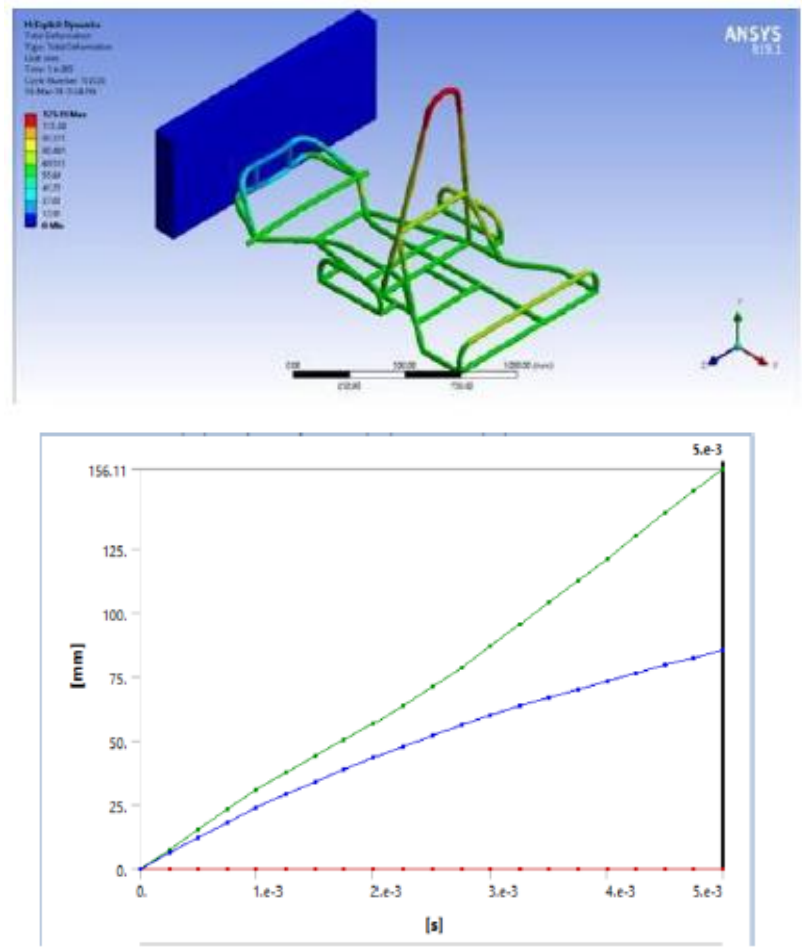

Figure-8 Total deformed chassis at $22 \mathrm{~m} / \mathrm{s}$.
Published By:

Blue Eyes Intelligence Engineering $\&$ Sciences Publication 
Impact Analysis on Go-Kart Chassis with Variable Speeds using Ansys 19.0

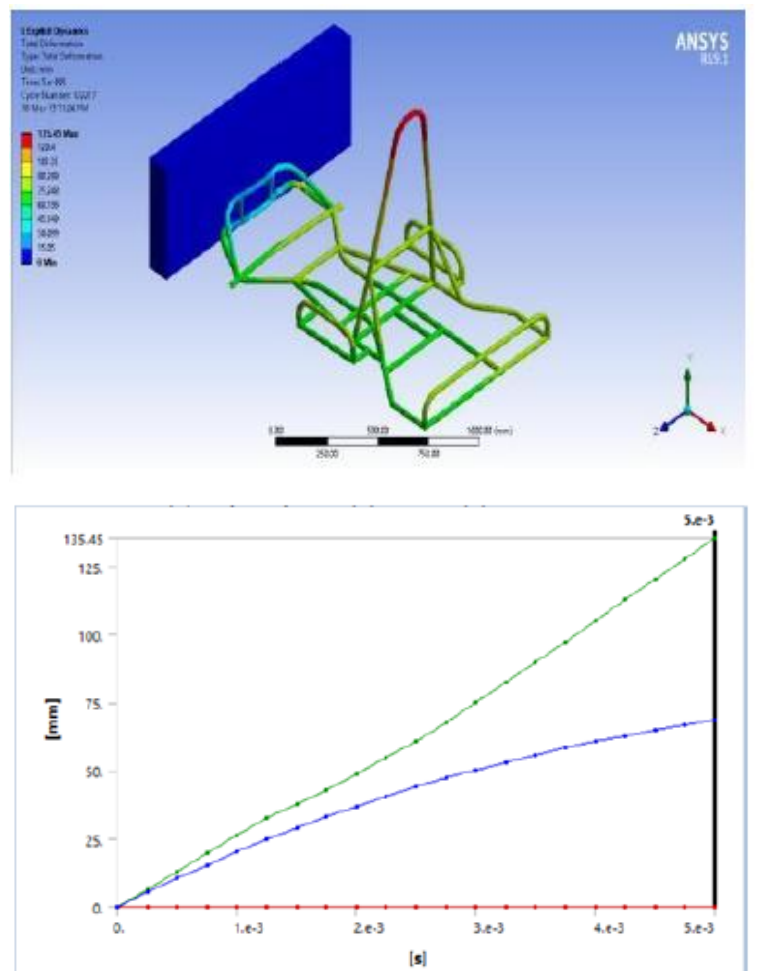

Figure-9 Total deformed chassis at $24 \mathrm{~m} / \mathrm{s}$.

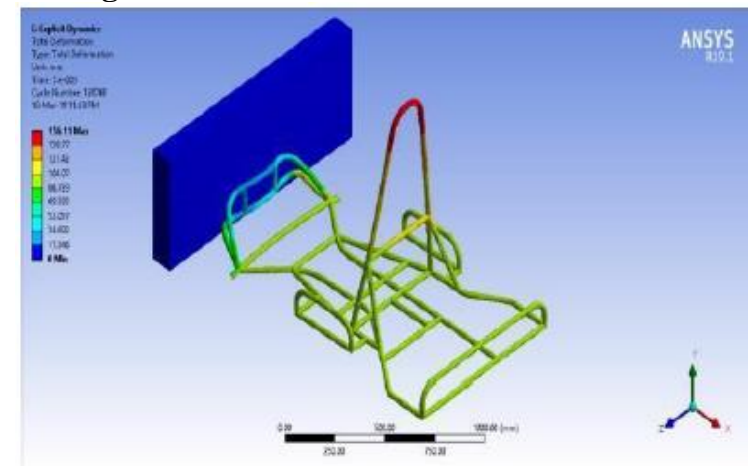

Figure-10 Total deformed chassis at $28 \mathrm{~m} / \mathrm{s}$.

Equivalent stress generated in the chassis at different speeds:
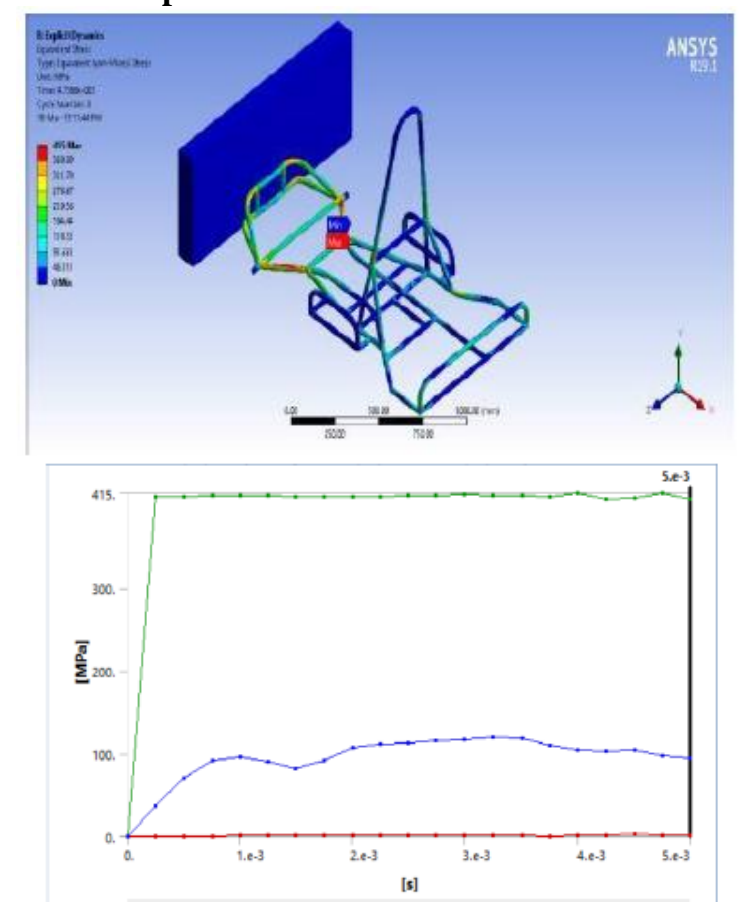

Figure-11 Equivalent stress at the speed of $18 \mathrm{~m} / \mathrm{s}$.

Retrieval Number F8494088619/2019@BEIESP

DOI: 10.35940/ijeat.F8494.088619

Journal Website: www.ijeat.org
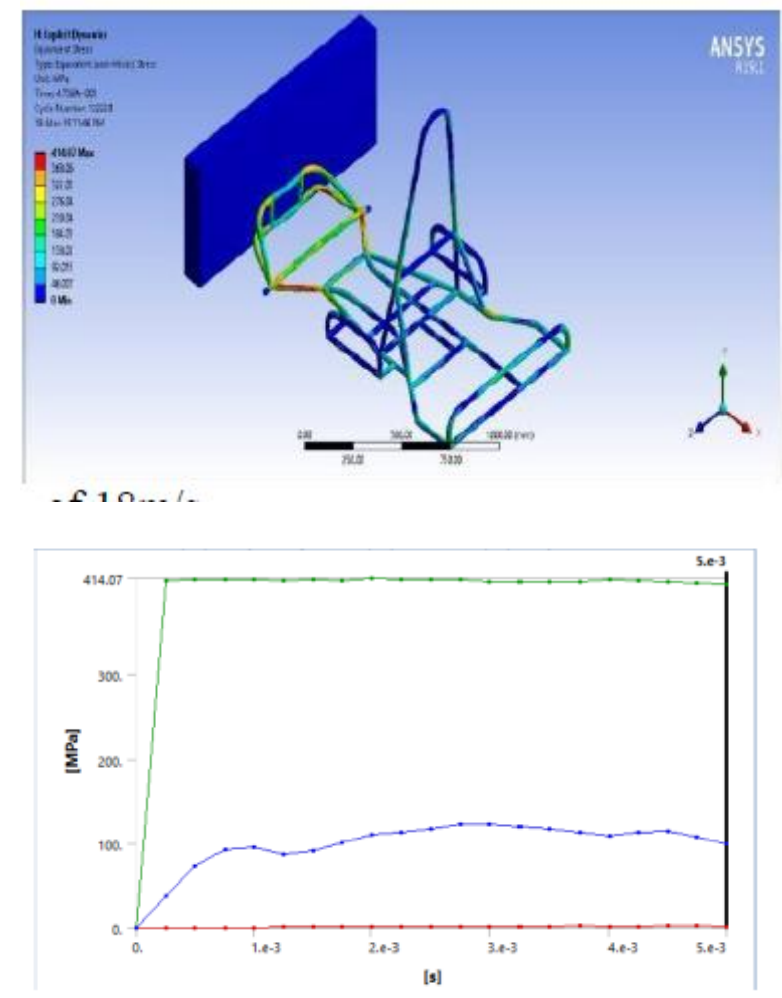

Fig12: Equivalent stress at the speedof $22 \mathrm{~m} / \mathrm{s}$.
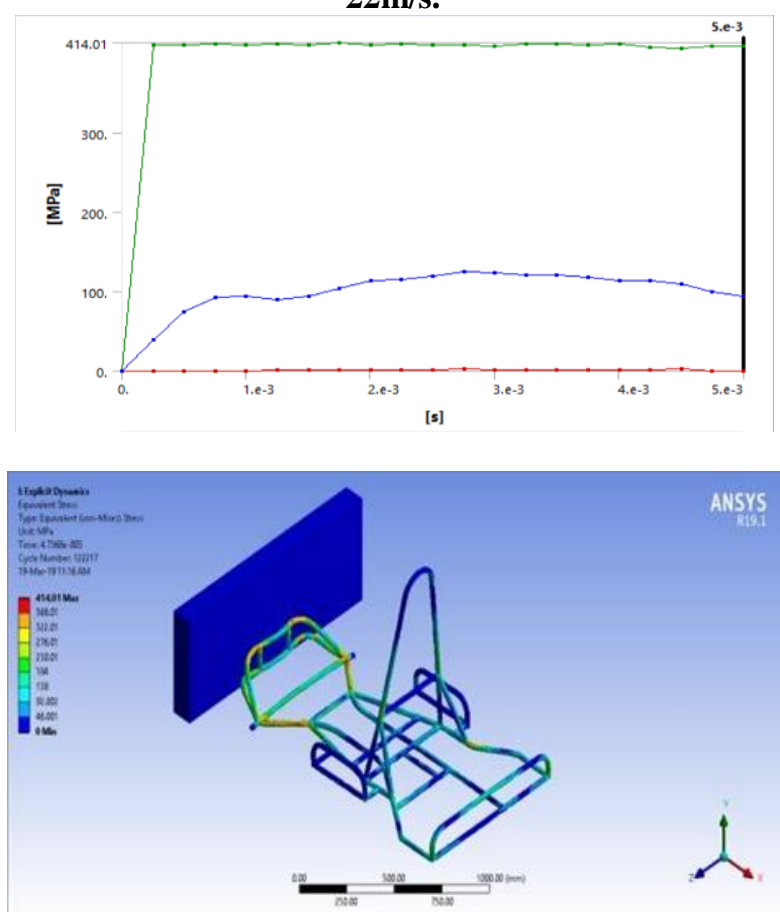

Figure-13 Equivalent stress at the speedof $24 \mathrm{~m} / \mathrm{s}$. 

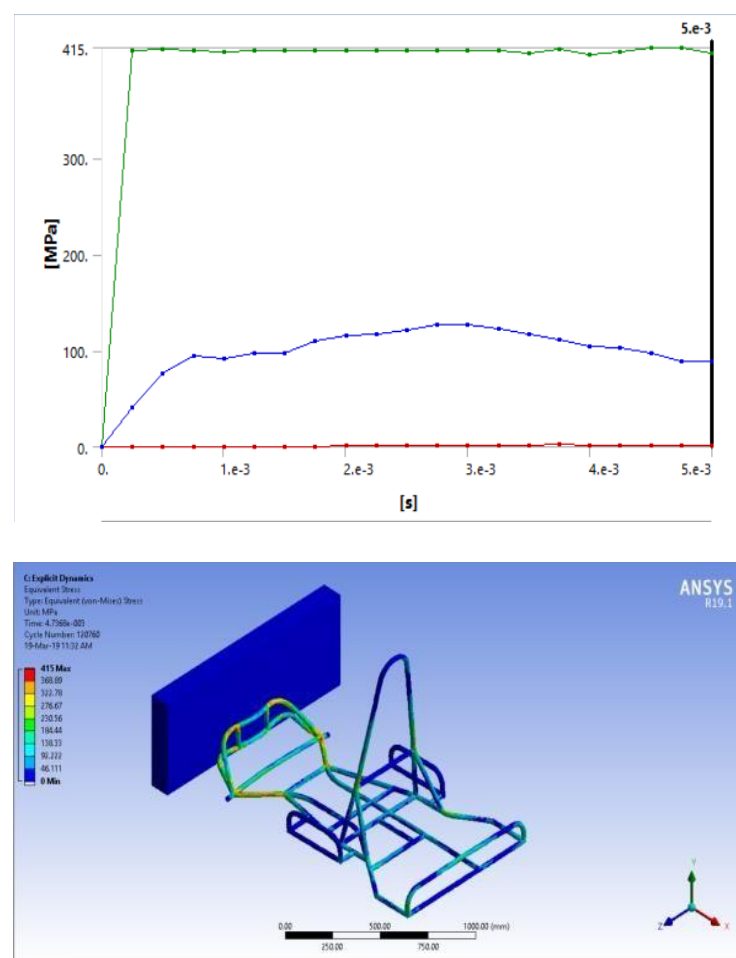

Figure-14 Equivalent stress at the speed of $28 \mathrm{~m} / \mathrm{s}$.

Equivalent Strain in the chassis atdifferent speeds.
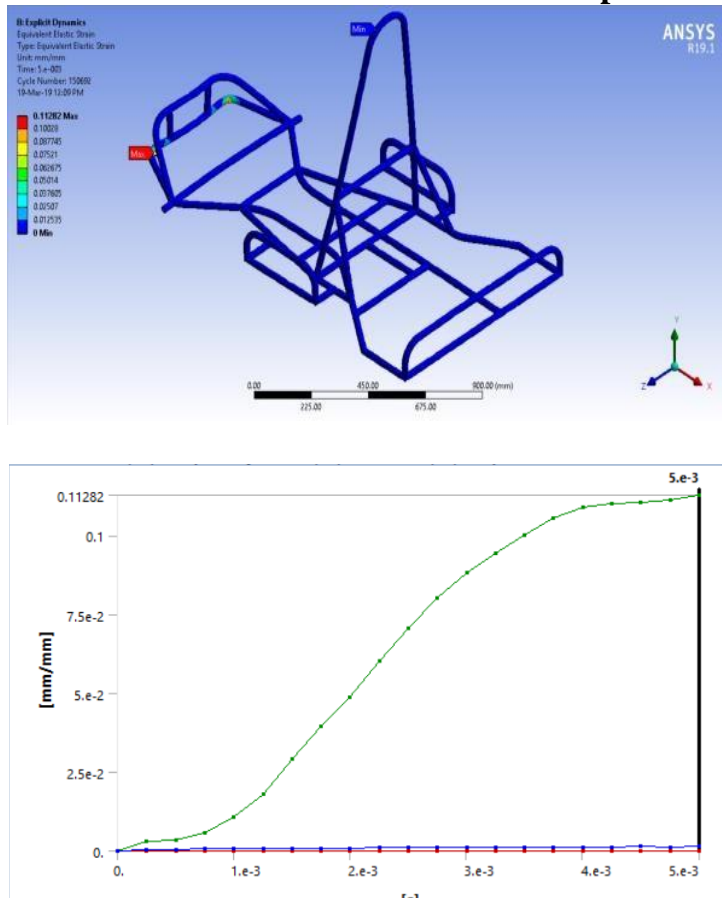

Figure-15 Equivalent strain at the speed of $18 \mathrm{~m} / \mathrm{s}$.

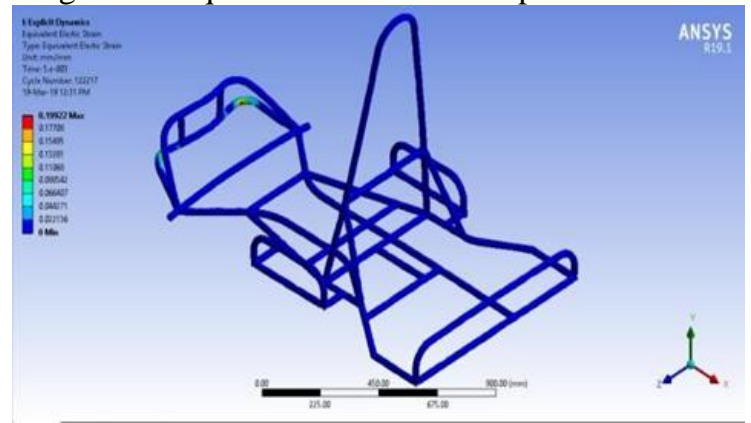

Retrieval Number F8494088619/2019@BEIESP

DOI: 10.35940/ijeat.F8494.088619

Journal Website: www.ijeat.org

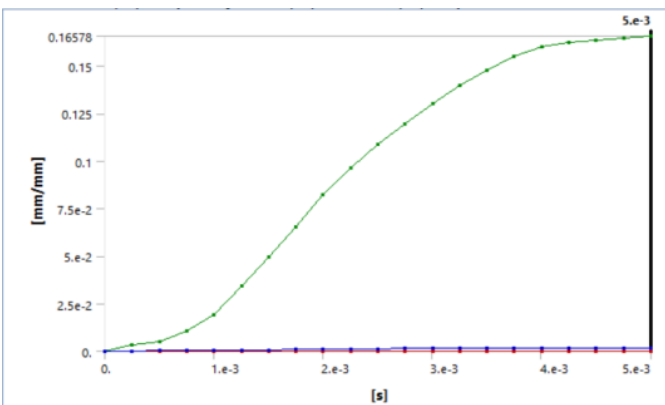

Figure-16 Equivalent strain at the speed of $22 \mathrm{~m} / \mathrm{s}$
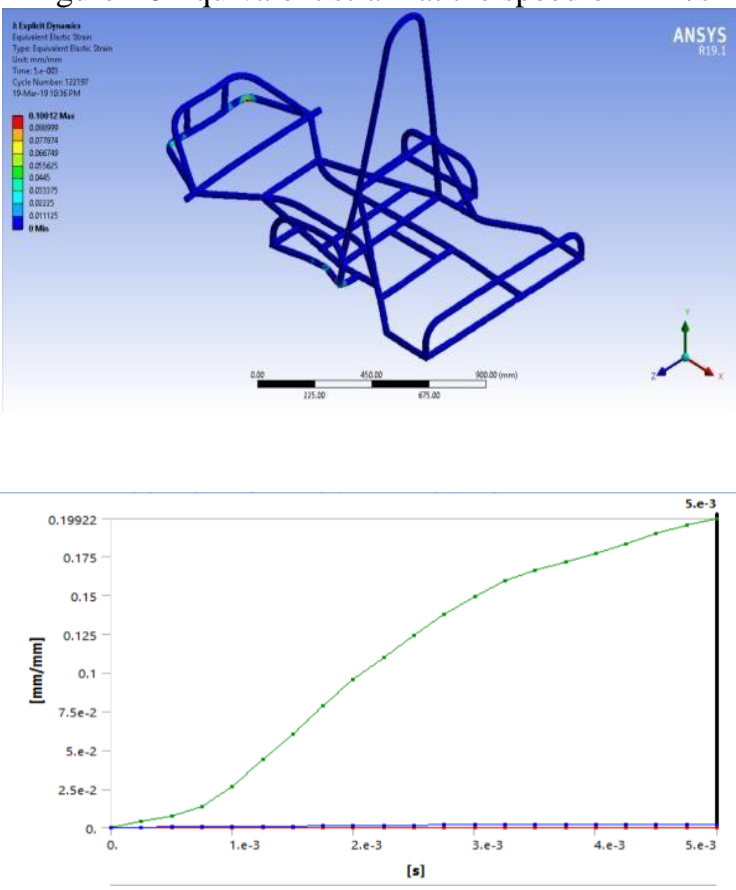

Figure-17 Equivalent strain at the speed of $24 \mathrm{~m} / \mathrm{s}$.
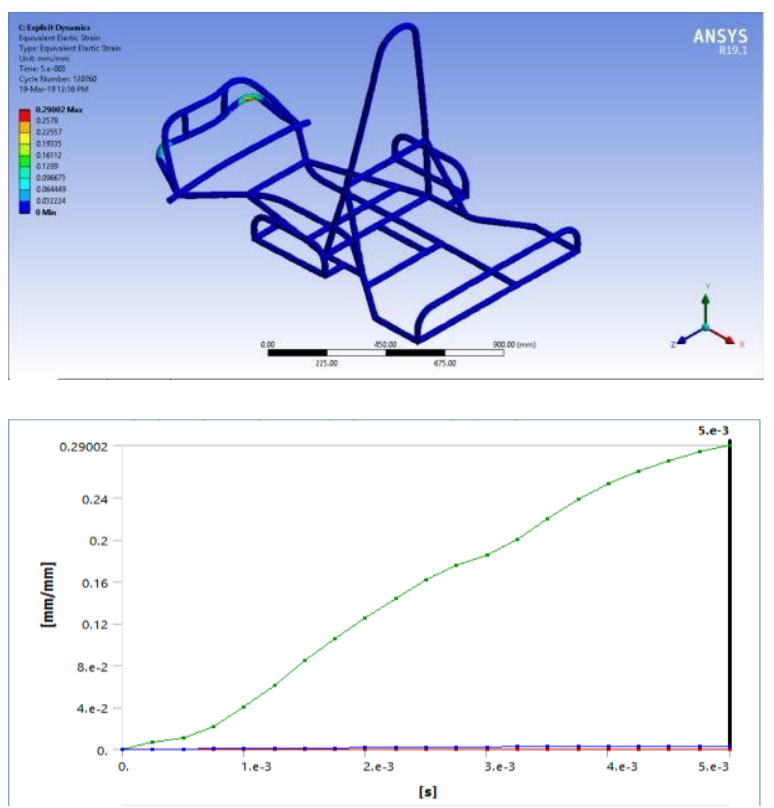

Figure-18 Equivalent strain at the speed of $28 \mathrm{~m} / \mathrm{s}$.

Published By:

Blue Eyes Intelligence Engineering \& Sciences Publication

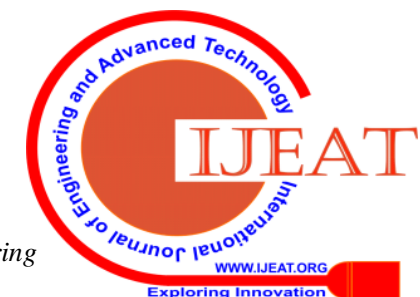


Impact Analysis on Go-Kart Chassis with Variable Speeds using Ansys 19.0

Total Deformation generated in the chassis:
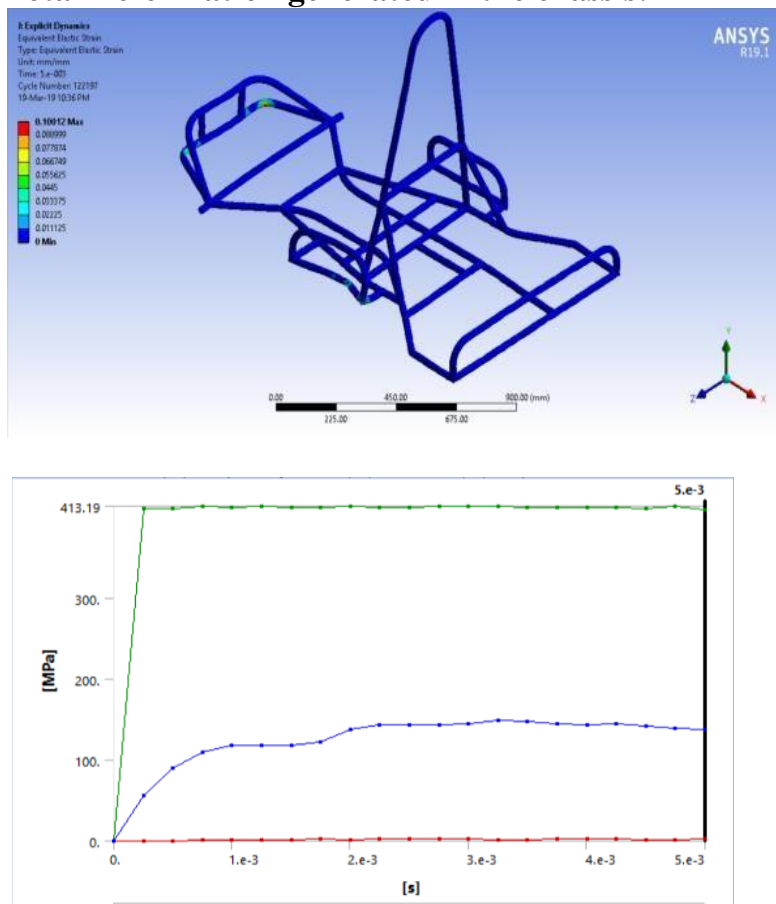

Figure-19 Total deformation at the speed of $18 \mathrm{~m} / \mathrm{s}$.

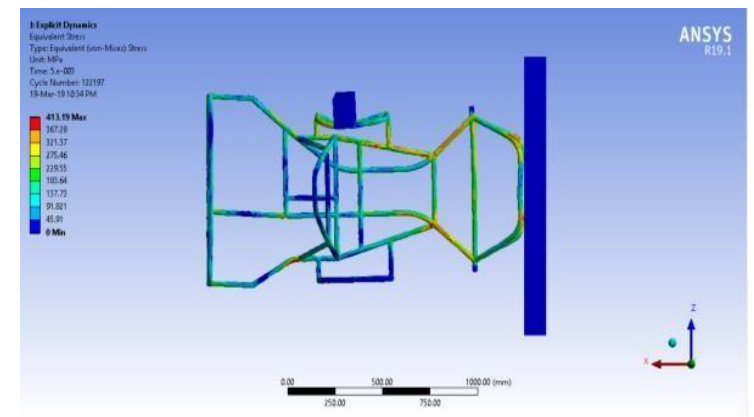

Figure 20: Equivalent stress at $18 \mathrm{~m} / \mathrm{s}$.

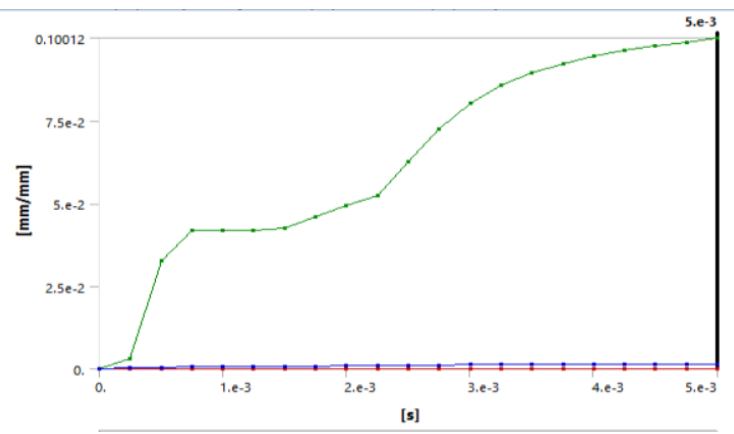

Figure-21 Equivalent strain at the speed of $18 \mathrm{~m} / \mathrm{s}$. Comparison Plots between Total Deformation, Equivalent Stress and Equivalent Strain with respect to speeds:
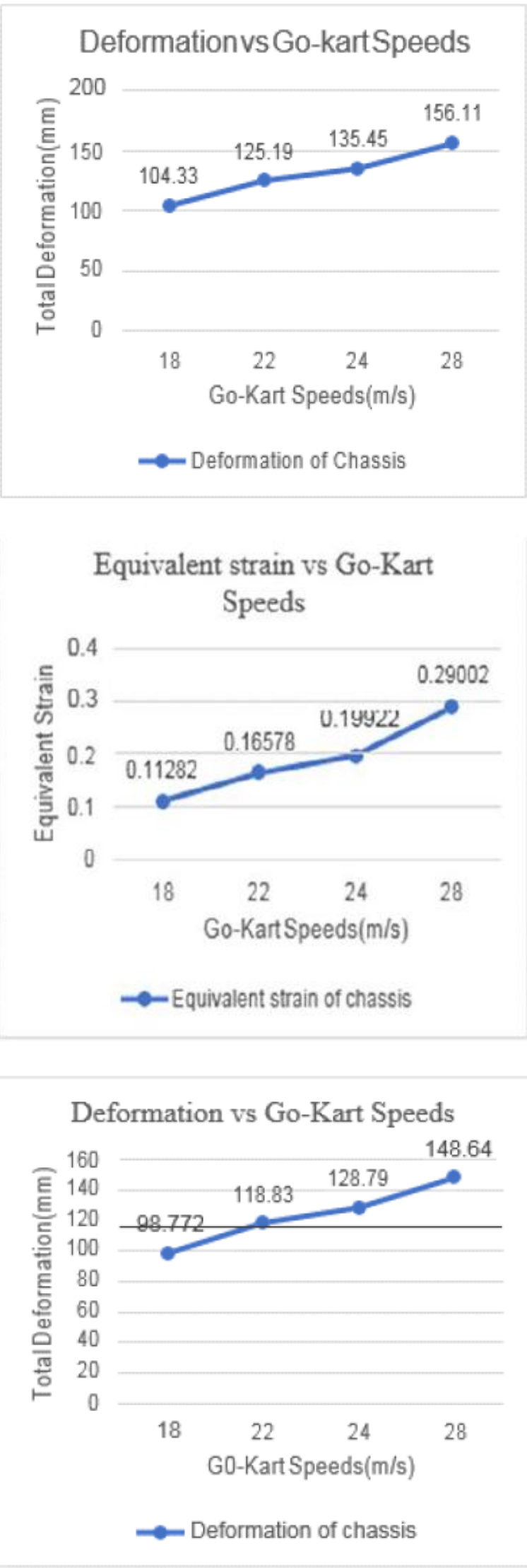

V. CONCLUSION:

The Go-kart chassis had been modelled in solid works and performed the impact analysis of the motor vehicle chassis in Ansys.

Published By: Blue Eyes Intelligence Engineering DOI: 10.35940/ijeat.F8494.088619 
The impact and behaviour of the chassis during impact on the go-kart chassis been analysed and noted. The basic requirements of the chassis like less weight and more strength are considered while doing analysis. The material that we opted for this chassis is AISI 4130 which is a medium carbon steel having good tensile strength and better machinability and offers good balance to toughness and ductility. Such that by performing impact analysis at different impact speeds we

have analysed the chassis behaviour for the given conditions. The given chassis is tested under different impact conditions and the results such as deformations, stresses are determined using ANSYS 19.0 software. we conclude that this design is satisfying all the safety conditions as well as ergonomically designed. This analysis can also be used for conventional Automobiles. Further optimizations can be achieved.

\section{REFERENCES:}

1. Trusses \& Arches by Charlsezragreene (schooler's choice)

2. Structural mechanics modeling and analysis of frames and trusses by KARL-GUNNAR

3. Automotive ergonomics by Nikolaos gkikas.

4. Ansys official webpage( $\underline{w w w . a n s y s . c o m ~})$

5. Encyclopedia.(https://www.wikipedia.org/)

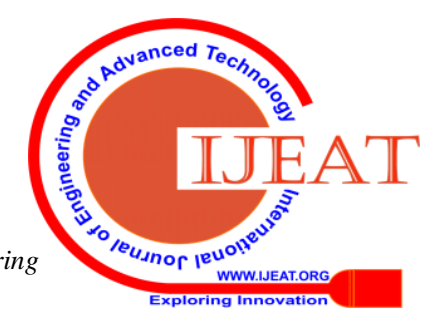

